\title{
Biomarkers in CVDs Prevention through Nutrition and Physical Activity
}

Vera Simovska-Jarevska*

Faculty of Technology and Technical Sciences Veles, Study of Nutrition, University St. Kliment Ohridski - Bitola, Macedonia

"Corresponding author: Vera Simovska-Jarevska, University St. Kliment Ohridski - Bitola, Faculty of Technology and Technical Sciences Veles, Study of Nutrition, Macedonia, Tel: 38923225402; E-mail: vera.simovska@uklo.edu.mk

Rec date: April 08, 2016; Acc date: April 11, 2016; Pub date: April 18, 2016

Copyright: (c) 2016 Simovska-Jarevska V. This is an open-access article distributed under the terms of the Creative Commons Attribution License, which permits unrestricted use, distribution, and reproduction in any medium, provided the original author and source are credited.

Citation: Jarevska VS (2016) Biomarkers in CVDs Prevention through Nutrition and Physical Activity. J Nutr Disorder Ther 6: e125. doi:10.4172/2161-0509.1000e125

\section{Editorial}

To understand how individually dosed and programmed physical activity in conjunction with moderate hyper-protein diet induces physiological cardiac growth using biomarker of myocyte proliferation is innovative approach in CVD prevention.

The main objective of our project is to support multidisciplinary research team that will use innovative and scientific approach to increase knowledge on the validation the roles of biomarkers, microRNAs (focused on MiR-17-3p) in exercise and specific dietinduced cardiomyocite proliferation.

Given the limited regenerative capacity of cardiac hypertrophy, it's unclear whether this results in prevention of CVDs. The roles of MicroRNAs in cardiac response to physical activity and nutrition are unknown. The clinical relevance of the circulating MicroRNAs (miR-17-3p) is confirmed with the fact that it's increased during physical activity in patients with heart diseases.

The specific circulating biomarker MicroRNAs (miR-17-3p) responses is identified during individually dosed, programmed physical activity and moderate hyper-protein diet in patients with heart failure. In this controlled clinical trial is included 73 subjects during the period of 3 months.
Numerous experimental studies have confirmed that factors responsible for physiological hypertrophy may be antagonist to development of pathological cardiomiopathy. Regular individually dosed, programmed physical activity and specific diet induces physiological cardiac growth including an increase in markers of myocyte proliferation.

The project includes a systematic exploration and validation of biomarkers to obtain a good coverage of the specific diet and physical activity in patients with initial phase of heart disease (for example high normal or moderate arterial hypertension) exploring myocardial effects using biomarker MicroRNAs (miR-17-3p) and biomarkers of food intake.

Understanding how individually dosed and programmed physical activity and specific diet have positive effects on cardiac growth may help to identify innovative therapeutically concept to prevent the CVDs as consequences of pathological stress. MicroRNAs (MiRNAs) are central regulators of gene expression and their deregulation can results in CVDs. 